\title{
Investigation of the Self-Injection Locked Behaviour of a Continuous Wave Nd:YAG Ring Laser
}

\author{
Tobias Meier, Benno Willke, Marina Dehne, and Karsten Danzmann \\ Albert Einstein Institute, Max Planck Institute for Gravitational Physics, and Institut für Gravitationsphysik, \\ Leibniz Universität Hannover, Callinstraße 38, D-30167 Hannover, Germany. \\ Author phone: +49-511-762-17170, fax: +49-511-762-2784, e-mail address: tobias.meier@aei.mpg.de
}

\begin{abstract}
External redirection of light from one propagation direction of a ring laser back into the opposite direction forces uni-directional operation. We investigated this so-called self-injection locking effect and achieved single-frequency operation.

(C) 2009 Optical Society of America

OCIS codes: (000.2190) Experimental physics; (140.3570) Lasers, single mode
\end{abstract}

\section{Introduction}

Applications in high precision optical metrology often require continuous wave lasers which emit with demanding stability at high power levels and in a single longitudinal and transversal mode. Solid-state ring lasers with active media like Nd:YAG are a good choice for this because of their beneficial intrinsic properties like homogenous line broadening, high efficiency, and wide tunability [1]. In order to decouple the high power generation from the high stability requirement often the injection locking technique is adopted to enforce uni-directional emission in a single longitudinal and transversal mode. On the one hand, this helps to abandon as many optical components from inside the laser resonator as possible which could degrade the lasers performance by their absorption, thermal lensing and stress-induced birefringence [2]. On the other hand a more complex system and further costs arise from the need for an extra master laser. Hence, finding a possibility to eliminate the master laser would be advantageous.

Another way to achieve single directional emission without additional elements inside the laser resonator is external feedback from one propagation direction of the laser back into the other, referred to as self-injection locking (SIL). Efforts have been made in the past to use this method to produce and influence oscillations of laser output power [3,4], to investigate nonlinearities and nonreciprocities inside the laser resonator [5], or to produce simultaneous multiple wavelength output from a single laser resonator [6].

In this work we investigate the capabilities of the self-injection locking effect with respect to the production of stable output power in a single transversal and single longitudinal mode, in order to test its feasibility as a replacement for standard continuous wave injection locked setups in high precision optical metrology.

\section{Experiment}

The laser system used is a copy of the GEO 600 slave laser [7]. It consists of a ring resonator in bow-tie configuration, two Nd:YAG rods of $6 \mathrm{~mm}$ length each and two fused silica plates placed under Brewster angle to introduce losses for the unwanted vertical polarization and to compensate for astigmatism (see inset of figure 1).

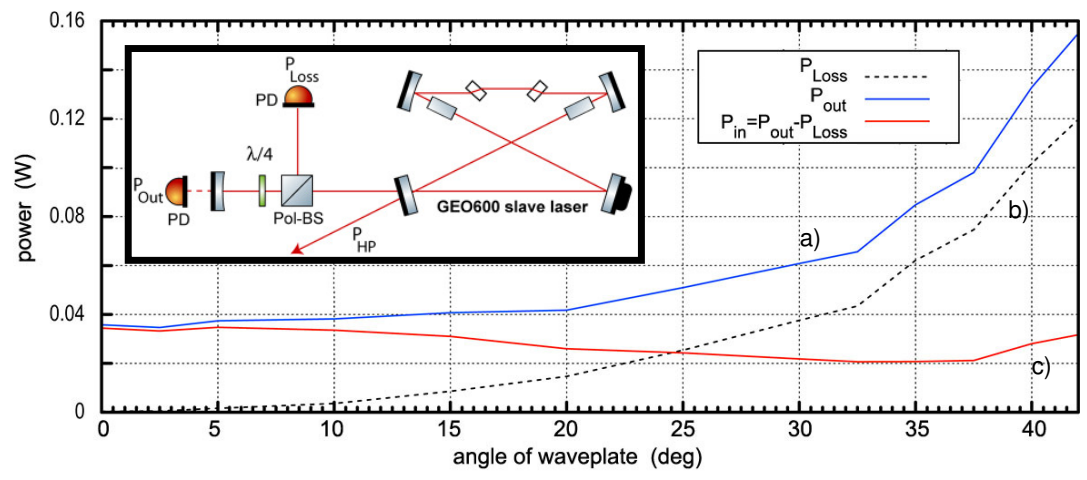

Fig. 1: Power emitted into the SIL arm $P_{\text {out }}$ (a), power split off by the beam splitter $P_{\text {Loss }}(b)$, and power reinjected into the laser $P_{\text {in }}$ (c) plotted as a function of losses in the SIL path. The output power

$\mathrm{P}_{\mathrm{HP}}$ was nearly constant for all shown waveplate angles. Inset: Corresponding experimental setup. 


\section{CTuP2.pdf}

In a preceded injection locked setup this slave laser produced approximately $12 \mathrm{~W}$ of output power in a linear polarized, single-mode, single-frequency operation. But when blocking the master laser, the slave laser changes from stable behaviour to strong full-scale oscillations comparable to those explained in [3], corresponding to an oscillation between the two possible propagation directions. In order to force the laser back into single directional operation we enhanced the gain of its one propagation direction (the high power mode) by backreflection of light from the other direction of propagation (SIL mode) with a curved mirror. Its radius of curvature and alignment was chosen to achieve good modematching with the high power mode (see inset of figure 1).

With this setup we achieved long-term stable single directional operation of the laser system with less than $1 \%$ of the output power in the SIL path $\left(\mathrm{P}_{\mathrm{out}}\right)$ and approximately $10 \mathrm{~W}$ of output power in the high power path $\left(\mathrm{P}_{\mathrm{HP}}\right)$. In this state the laser emits its high power mode linearly polarized and with a good Gaussian beam shape. A measurement of its longitudinal mode structure, however, shows that the laser does not operate on a single longitudinal mode but emits simultaneously at multiple frequencies spaced by the lasers free spectral range (FSR).

Using a combination of a polarizing beam splitter and a quarter-waveplate to vary the losses in the SIL path reveals that the SIL mode greatly compensates for losses introduced in the feedback for the high power mode maintaining the stable single-directional operation over a wide range of losses up to 75\% (see main part of figure 1). This behaviour indicates that the two propagation directions are coupled. We exploit this coupling by introducing an additional low finesse ring resonator into the SIL path, locking it to the light of the SIL mode and investigating the longitudinal mode structure of the high power mode by use of a Scanning-FPI as a spectral analyzer (figure 2).

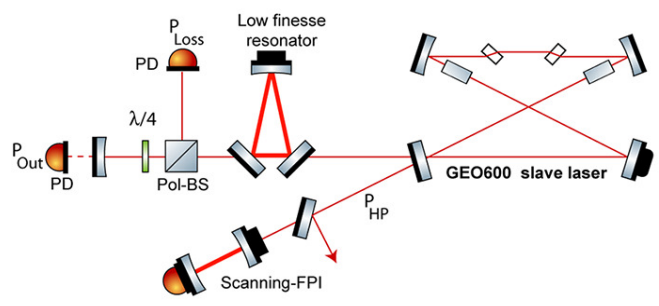

Fig. 2: Experimental setup for achieving single frequency operation of the high power mode.

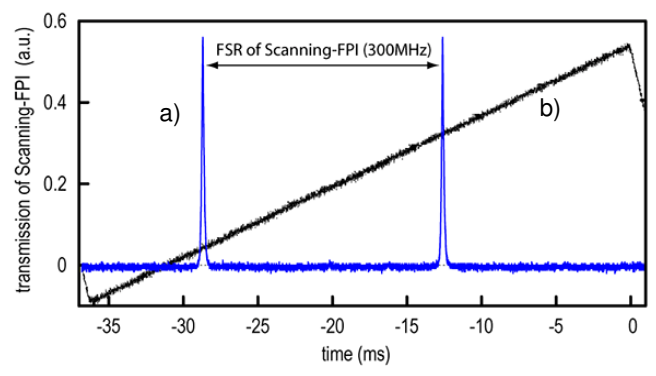

Fig. 3: Transmission of the Scanning-FPI of figure 2. Trace a) shows two successive longitudinal modes of the FPI with no other frequency components in between representing single frequency operation of the high power mode. Trace b) shows the FPI's ramp.

The external low finesse resonator selects a single longitudinal mode from the light of the SIL mode. This narrows the feedback for the high power mode to the selected frequency component, which causes the lasers high power beam to switch to linearly polarized single frequency operation. Figure 3 shows a scan of the Scanning-FPI that receives some light of the high power beam. The spacing of the transmission peaks corresponds to one FSR of the Scanning-FPI. This indicates single frequency operation of the lasers high power mode because laser light at multiple longitudinal modes would show up as additional peaks within one FSR of the FPI.

\section{References}

[1] W. Demtröder "Laser Spectroscopy, third edition," Springer-Verlag Berlin Heidelberg, 2003, chapter 5.

[2] M. Frede, R. Wilhelm, M. Brendel, C. Fallnich, F. Seifert, B. Willke, and K. Danzmann "High power fundamental mode Nd:YAG laser with efficient birefringence compensation," Optics Express 12 (15), 3581-3589 (2004).

[3] I.I. Zolotoverkh, N.V. Kravtsov, E.G. Lariontsev, and A.A. Makarov "Interaction of self-modulation and relaxation oscillations, and its role in nonlinear dynamics of a solid-state ring laser," Quantum Electronics 27 (7), 621-625 (1997).

[4] N.V. Kravtsov, N.N. Kravtsov, A.A. Makarov, and V.V. Firsov "Operational regimes of a ring chip laser with asymmetric coupling of counterpropagating waves,” Quantum Electronics 26 (3), 189-190 (1996).

[5] N.V. Kravtsov, and E.G. Lariontsev "Nonlinear dynamics of solid-state ring lasers," Quantum Electronics 36 (3), 192-221 (2006).

[6] Y. Louyer, J.P. Wallerand, M. Himbert, M. Deneva, and M. Nenchev "Two-wavelength, passive self-injection-controlled operation of diodepumped cw Yb-doped crystal lasers,” Applied Optics 42 (27), 5463-5476 (2003).

[7] I. Zawischa, M. Brendel, K. Danzmann, C. Fallnich, M. Heurs, S. Nagano, V. Quetschke, H. Welling, and B. Willke "The GEO 600 laser system," Classical and Quantum Gravity 19, 1775-1781 (2002). 